\title{
Certification of UK gastrointestinal endoscopists and variations between trainee specialties: results from the JETS e-portfolio
}

(요 $\odot \ominus$

\author{
Authors \\ Keith Siau1,2,3, John T. Anderson ${ }^{1,4}$, Roland Valori ${ }^{1,4}$, Mark Feeney ${ }^{1,5}$, Neil D. Hawkes ${ }^{1,6}$, Gavin Johnson ${ }^{1,7}$, Brian C. \\ McKaig $^{1,8}$, Rupert D. Pullan ${ }^{9}$, James Hodson ${ }^{10}$, Christopher Wells ${ }^{1,11}$, Siwan Thomas-Gibson ${ }^{1,12,13}$, Adam V. \\ Haycock $^{1,12,13}$, Ian L.P. Beales ${ }^{1,14}$, Raphael Broughton ${ }^{1}$, Paul Dunckley ${ }^{1,4}$, on behalf of the Joint Advisory Group on \\ Gastrointestinal Endoscopy (JAG)
}

Institutions

1 Joint Advisory Group on Gastrointestinal Endoscopy, Royal College of Physicians, London, UK

2 Department of Endoscopy, Dudley Group Hospitals NHSFT, Dudley, UK

3 Medical and Dental Sciences, University of Birmingham, Birmingham, UK

4 Department of Gastroenterology, Gloucestershire Hospitals NHSFT, Gloucester, UK

5 Department of Gastroenterology, Torbay and South Devon NHS Foundation Trust, Torquay, UK

6 Department of Gastroenterology, Cwm Taf University Health Board, Llantrisant, UK

7 Department of Gastroenterology, University College London Hospitals NHSFT, London, UK

8 Department of Gastroenterology, Royal Wolverhampton Hospitals NHS Trust, Wolverhampton, UK

9 General and Colorectal Surgery, Torbay and South Devon NHS Foundation Trust, Torquay, UK

10 Institute of Translational Medicine, University Hospitals Birmingham, Birmingham, UK

11 Department of Gastroenterology, North Tees and Hartlepool NHS Foundation Trust, Stockton on Tees, UK

12 Wolfson Unit for Endoscopy, St Mark's Hospital, London, UK

13 Imperial College London, London, UK

14 Department of Gastroenterology, Norfolk and Norwich University Hospital, Norwich, UK

submitted 21.6.2018

accepted after revision $\quad 2.1 .2019$

\# Appendix Table 1-4

Online content viewable at:

https://doi.org/10.1055/a-0839-4476
Bibliography

DOI https://doi.org/10.1055/a-0839-4476 |

Endoscopy International Open 2019; 07: E551-E560

(c) Georg Thieme Verlag KG Stuttgart · New York

ISSN 2364-3722

Corresponding author

Dr. Keith Siau, Dudley Group of Hospitals NHS Foundation

Trust, Department of Gastroenterology, Pensnett Road

Dudley DY1 2HQ, United Kingdom of Great Britain and

Northern Ireland

Fax: +01384 244262

keithsiau@nhs.net

\section{ABSTRACT}

Introduction In the UK, endoscopy certification is administered by the Joint Advisory Group on Gastrointestinal Endoscopy (JAG). Since 2011, certification for upper and lower gastrointestinal endoscopy has been awarded via a national (JETS) e-portfolio to the main training specialties of: gastroenterology, gastrointestinal surgeons (GS) and non-medical endoscopists (NME). Trends in endoscopy certification and differences between trainee specialties were analyzed.

Methods This prospective UK-wide observational study identified trainees awarded gastroscopy, sigmoidoscopy, colonoscopy (provisional and full) certification between June 2011-2017. Trends in certification, procedures and time-to-certification, and key performance indicators (KPIs) in the 3-month pre- and post-certification period were compared between the three main training specialties.

Results Three thousand one hundred fifty-seven endoscopy-related certifications were awarded to 1928 trainees from gastroenterology (52.3\%), GS (28.4\%) and NME (16.5 $\%)$ specialties. During the study period, certification numbers increased for all modalities and specialties, particularly NME trainees. For gastroscopy and colonoscopy, procedures-to-certification were lowest for GS $(P<0.001)$, whereas time-to-certification was consistently shortest in 
NMEs $(P<0.001)$. A post-certification reduction in mean cecal intubation rate $(95.2 \%$ to $93.8 \%, P<0.001)$ was observed in colonoscopy, and D2 intubation ( $97.6 \%$ to $96.2 \%$, $P<0.001)$ and J-maneuver $(97.3 \%$ to $95.8 \%, P<0.001)$ in gastroscopy. Overall, average pre- and post-certification KPIs still exceeded national minimum standards. There was an increase in PDR for NMEs after provisional colonoscopy certification but a decrease in PDR for GS trainees after sigmoidoscopy and full colonoscopy certification.

Conclusion Despite variations among trainee specialties, average pre- and post-certification KPIs for certified trainees met national standards, suggesting that JAG certification is a transparent benchmark which adequately safeguards competency in endoscopy training.

\section{Introduction}

It is desirable to have a process for determining whether an individual is competent to perform an endoscopic procedure before that individual is allowed to practice independently. This helps reassure and protect both patients and the service. Ideally the process should be identical regardless of the type of health professional seeking certification. Performance following certification should meet current performance standards for each procedure performed and ideally be subjected to ongoing audit and monitoring [1].

In the UK, a certification process exists to ensure that trainees in endoscopy have achieved a minimum standard of competence before being allowed to practice independently. This process is applied to all health professionals training in endoscopy and is governed by the Joint Advisory Group on Gastrointestinal Endoscopy (JAG) [2]. Since June 2011, the process has been administered through the JETS (JAG Endoscopy Training System) e-portfolio [3] and certification awarded electronically (e-certification) for gastroscopy [4], sigmoidoscopy [5], and colonoscopy [6]. This evolved from paper-based portfolios which were cumbersome, time-consuming and inconsistently completed [7]. All endoscopy training units are linked to the JETS e-portfolio which allows an individual trainee to enter training data from any JAG-registered training unit during their training rotation. The JETS e-portfolio enables trainees to record details of training episodes to generate procedure-specific key performance indicators (KPIs). Trainers use the same platform to complete competency assessments, i.e. Direct Observation of Procedural Skills (DOPS) and Polypectomy Skills (DOPyS), at regular intervals to monitor skills progression [8]. Input of assessments from multiple different trainers increases the reliability of DOPS [9] and DOPyS assessments [10]. The JAG recommends that endoscopy training be delivered at accredited endoscopy units by JAG-certified trainers $[7,11]$. The JETS eportfolio currently contains more than 2 million trainee procedural records and is unique to the UK.

The JETS e-portfolio has a procedure-specific checklist that informs trainees when they are ready for a summative assessment and can apply for certification (Appendix Table 1, Table 2, Table 3, Table 4) [3]. These include: attainment of minimum procedural numbers and procedure-specific key performance indicators (KPIs), attendance of training courses, and satisfactory completion of DOPS and DOPyS [12-14]. Once these criteria are satisfied, trainees may apply for summative assessment. For certification in gastroscopy and sigmoidoscopy, the process is entirely formative, until the summative assessment is undertaken. Colonoscopy certification differs and is currently awarded in two phases: provisional and full certification (Appendix Table4). Once provisional colonoscopy certification status is achieved, endoscopists are allowed to practice diagnostic colonoscopy independently, but are subject to departmental supervision and monitoring of KPIs. [15] Trainees are able to perform sigmoidoscopy independently at this stage. Full colonoscopy certification is dependent on achieving provisional certification and additional criteria (Appendix Table4). No further summative assessment of colonoscopy skills is required to progress from provisional to full certification. The final stage of JAG certification requires that a local endoscopy training lead validate the data and sign off on the application for certification in the JETS e-portfolio. The application is then submitted electronically to JAG. Independent JETS assessors then review the application and decide whether certification is to be awarded [4-6].

In the UK, the endoscopy workforce consists predominantly of gastroenterologists, gastroenterology surgeons (GS), and non-medical endoscopists (NME), with radiologists and general practitioners forming a small minority (<2.5\%) [16]. Irrespective of specialty, all UK endoscopy trainees are required to meet the same procedural standards and go through the same standardized e-certification process. In recent years, demand for endoscopy in the UK has increased, requiring an increase in both endoscopy capacity and workforce [17]. In response, strategic recruitment initiatives such as the NME training program, backed by the Department of Health, have been successful in increasing numbers of NMEs in the workforce [18]. It is possible that this may have adversely impacted on the training list availability for other trainees and potentially, the time required to achieve certification.

There is no published literature on endoscopy certification within the UK or elsewhere. Trends in certification numbers have not been studied. Whether the UK certification process ensures that trainees reach acceptable standards for independent practice is unknown. It is important to determine whether the certification process is effective and to identify factors that affect how and when a trainee completes the certification process. This would inform regional and national training programs by allowing them to predict how rapidly a new workforce can be developed as demand for endoscopy changes. Moreover, such data could be used to optimize the existing training pathway, using evidence-based training interventions, to provide maximum efficiency in training an effective workforce. 
In this study we aimed to assess whether certification ensures trainees are adequately trained for independent practice by exploring pre- and post-certification KPIs, and explored how specialties compared with regard to trends in certification.

\section{Methods}

\section{Study Design}

This was a 6-year prospective, UK-wide, observational study. The study cohort consisted of all trainees who were awarded certification for gastroscopy, flexible sigmoidoscopy, and colonoscopy between 1st June 2011 and 1st June 2017 via the JETS e-portfolio. Data extracted included: trainee specialty, trainee level, date of certification, and procedure-specific KPIs.

In addition, lifetime procedure counts prior to certification were also collated for each trainee. For those who commenced training prior to inception of the e-certification system or outside the UK, procedures performed prior to JETS were not electronically recorded. As such, at the point of registration, these trainees recorded the numbers of procedures of each type that they had already performed, which is referred to as the baseline procedural count. These were added to the numbers of procedures recorded in the system, to give the lifetime procedural count.

\section{Outcomes}

For each certification modality and trainee specialty the following were reported:

1. Number of trainees awarded certification per year.

2. Lifetime procedure counts prior to successful certification.

3. Time taken from first procedure-specific JETS e-portfolio entry to certification. Trainees with baseline procedures prior to enrollment in the JETS e-portfolio were excluded from analysis of this outcome, as the dates of the first procedure were not recorded electronically.

4. KPIs in the 3 months before and 3 months after certification. These comprised: unassisted cecal intubation rate (CIR) and polyp detection rate (PDR) for colonoscopy, PDR for sigmoidoscopy, and rates of D2 intubation and J-maneuver for gastroscopy (retroflexion in the gastric antrum to visualise the cardia and gastric fundus). The CIR was unadjusted (not adjusted for factors such as poor-quality bowel preparation or impassable stricture). Trainees do not train on colorectal cancer screening procedures and are advised to exclude rectal hyperplastic polyps from the PDR. Trainees with fewer than 10 procedure-specific e-portfolio entries in either of these 3-month periods were excluded, to allow for reliable estimates of rates.

5. Rates and reasons for failed certification applications.

\section{Statistical analyses}

The numbers of certifications awarded in each annual period (June to May) were collated, and linear regression models were produced to quantify changes over time for each procedure type, and within each specialty. Lifetime procedure counts and times taken to achieve certification were then compared between specialties using Kruskal-Wallis tests, followed by pair- wise Dunn's post-hoc tests where significant differences were detected. Changes in KPIs between the 3-month pre- and post-certification periods were assessed using Wilcoxon's signed rank tests, with comparisons across specialties performed using Kruskal-Wallis tests, followed by Dunn's test. Rates of successful JAG applications were compared between specialties using chi $^{2}$ tests. All analyses were performed using SPSS (v23, Illinois, United States), with $P<0.05$ indicative of significance throughout.

\section{Baseline characteristics and trends in certification over time}

Between June 2011 and 2017, JAG awarded 3157 endoscopyrelated certifications across the four endoscopic modalities to 1928 trainees. The number of trainees awarded certification increased over time ( $>$ Fig.1). Gastroscopy was the modality for which the most certifications were awarded $(N=1312,41.6 \%)$, followed by provisional $(\mathrm{N}=1105,35.0 \%$ ) and full $(\mathrm{N}=546$, $17.3 \%$ ) colonoscopies, and sigmoidoscopy ( $N=194,6.1 \%)$. Numbers of certifications awarded per year increased significantly for all of the modalities by similar absolute rates, ranging from an additional 13 per year in sigmoidoscopy to 32 per year in provisional colonoscopy ( $>$ Fig. 1). However, when considered as relative increases, sigmoidoscopy and full colonoscopy had the greatest rate of change over the period, with the number of certifications awarded per year increasing 19-fold ( $N=75$ vs. 4) and 14-fold ( $N=152$ vs. 11), respectively, between 2011 12 and 2016- 17 (compared to 4-fold in provisional colonoscopy and 3 -fold in gastroscopy).

Trends in certifications are broken down by specialty in $\mathbf{T a}$ Table 1. Gastroscopy and both provisional and full colonoscopy certification were predominantly awarded to gastroenterology trainees, followed by GS and NME. While the gastroenterology specialty was the main contributor to the increasing number of colonoscopy certifications awarded per year in absolute terms, trends over time observed in this group were actually the least significant as, due to the large number of gastroenterologists awarded certification early in the period, the relative changes were small. For gastroscopy, the numbers of certifications awarded per year to gastroenterologists was not found to change significantly over time $(P=0.403, N=147$ in 2012/13 vs. 134 in 2016/17), suggesting that this has plateaued. Sigmoidoscopy certification was predominantly awarded to NMEs ( $N=148,76.2 \%)$ and, as such, these were the main cause of the observed yearly increase in certification.

\section{Procedural count and formative assessments before successful certification}

The median number (interquartile range [IQR]) of lifetime procedures recorded prior to certification were: gastroscopy: 282 (IQR 237-411), sigmoidoscopy: 262 (IQR 210-334), provisional colonoscopy certification: 269 (IQR 226-342) and full colonoscopy certification: 403 (IQR 339-509). The median number required to apply and achieve certification varied significantly by specialty in gastroscopy and provisional colonoscopy $(P<0.001)$ in the order of gastroenterology $>$ NME $>$ GS ( $\triangleright$ Fig.2). For full colonoscopy certification, the order was 


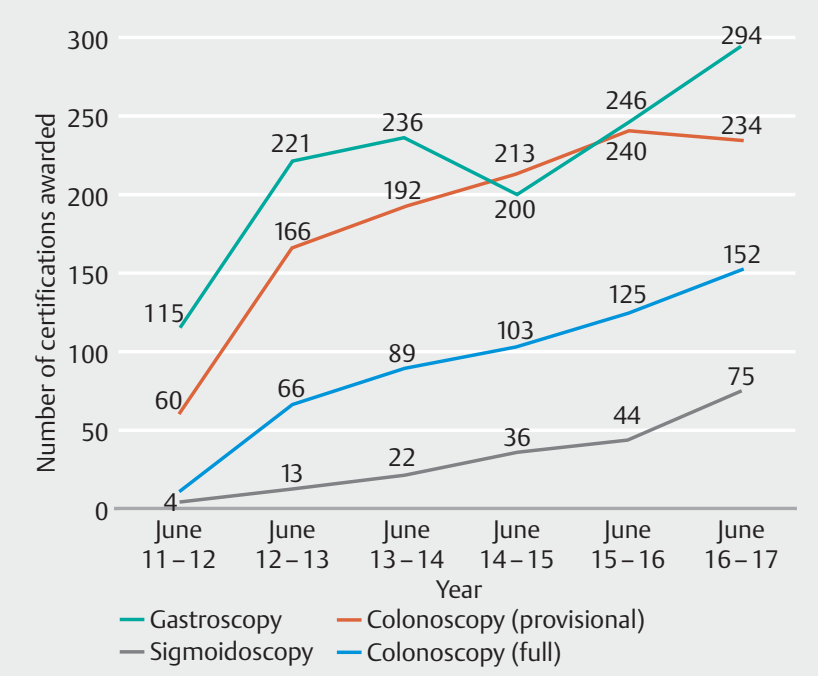

- Fig.1 Endoscopy certification awarded by year and modality.

NME $>$ gastroenterology $>$ GS $(P<0.001)$, while no significant difference between specialties was observed for sigmoidoscopy $(P=0.386)$.

There was significant variation in the number of precertification DOPS and DOPyS assessments performed between specialties $(P<0.001)$. NMEs had the greatest median number of formative assessments completed by trainers (30 DOPS, 9 DOPyS), followed by GI (29 DOPS, 8 DOPyS) and GS (18 DOPS, 8 DOPyS).

\section{Time-to-certification}

For each trainee, time-to-certification was measured using the interval between first procedure-specific e-portfolio entry and certification date. The analysis was restricted to $62.9 \%$ of trainees who reported no baseline procedures outside the JETS e-portfolio. For all specialties, median training times to certification were: gastroscopy 1.9y (IQR 1.2-3.0y); sigmoidoscopy: 1.6y (IQR 1.0-2.7y); provisional colonoscopy: 3.2y (IQR 2.2-4.5y) and full colonoscopy: 4.1y (IQR 2.8-5.2y). Training time to certification differed significantly across the three major specialties for each modality $(P<0.001)$ in the order of GS > gastroenterology $>$ NME, except for sigmoidoscopy, which was in the order of gastroenterology $>$ GS $>$ NME ( Fig. 3).

\section{Key performance Indicators}

\section{Gastroscopy}

A total of 768 trainees (58.5\%) met the inclusion criteria of $\geq 10$ post-certification procedures in the 3 months pre- and post-certification and were included in the analysis ( $\triangleright \mathrm{Ta}$ ble 2). Over this period, trainees recorded a median of 47 procedures (IQR 31 - 70) pre- and 34 (IQR 22-55) post-certification. Pre-certification, rates of D2 intubation (mean 97.6\%) and J-maneuver (mean $97.3 \%$ ) exceeded the JAG certification requirements of $\geq 95 \%$ for both KPIs (Appendix Table 1) [12].

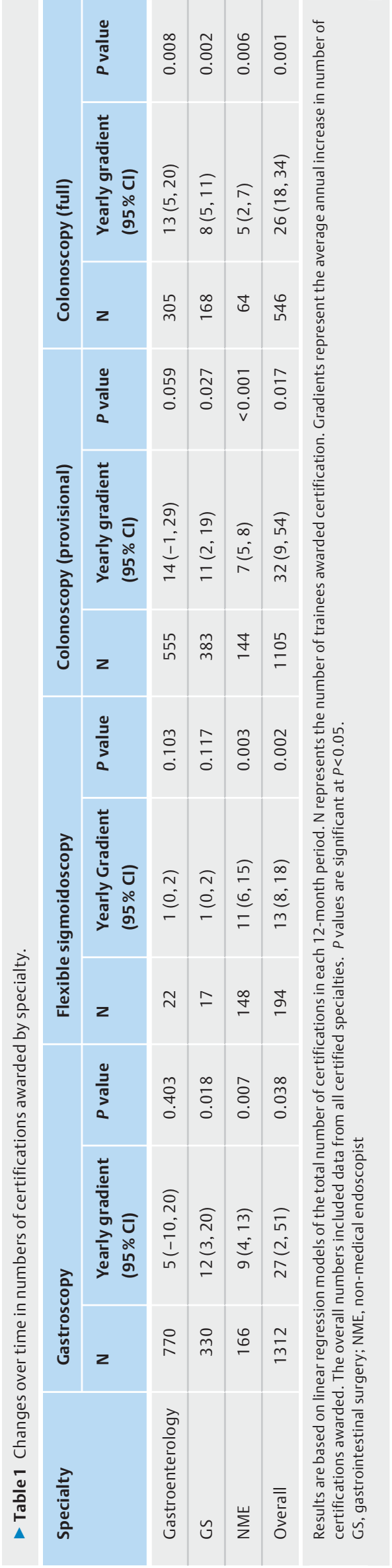




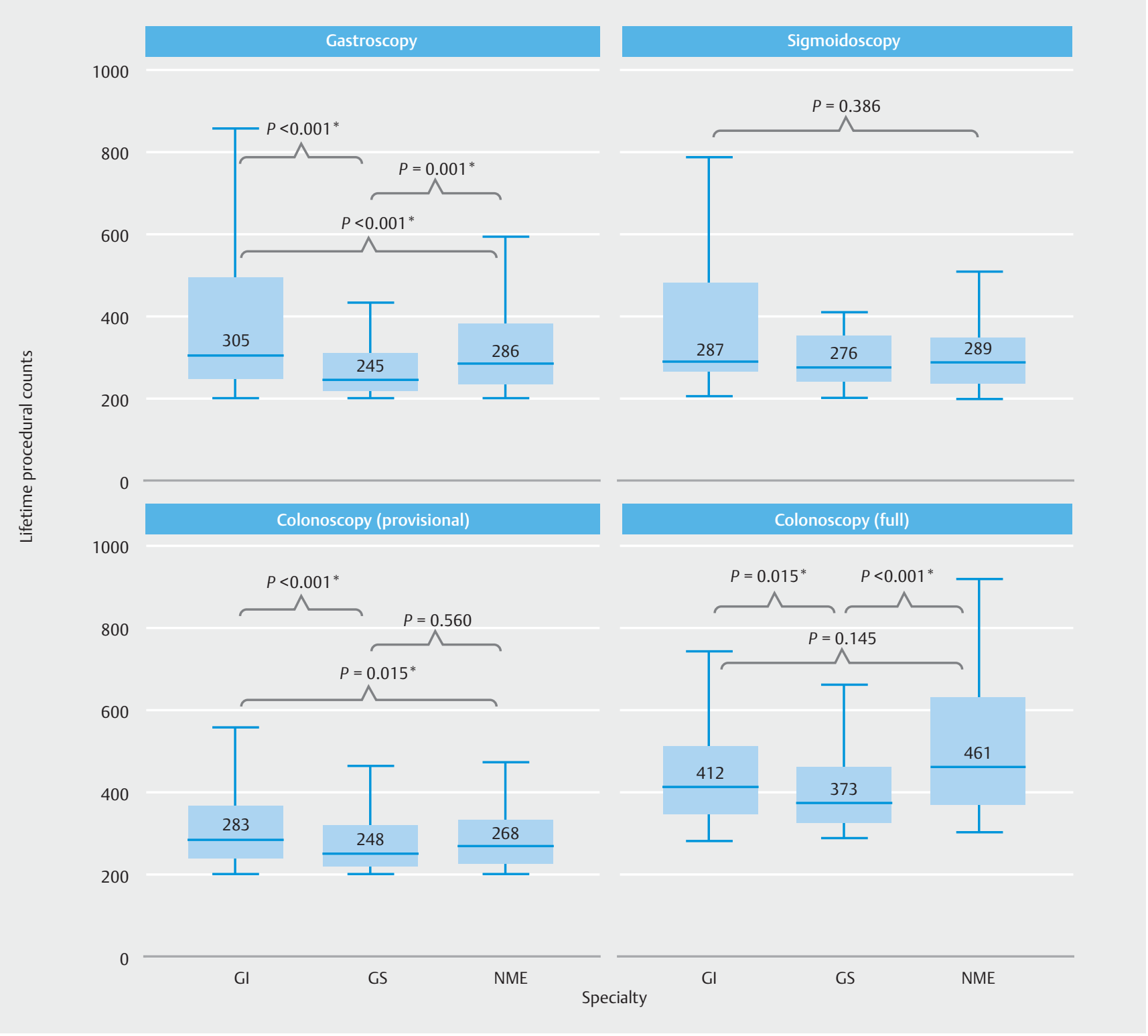

Fig. 2 Variations in precertification procedural counts by specialty trainees and certification modality. GS, general surgeon; NME: nonmedical endoscopist. ${ }^{*} P<0.05$.

Following certification, minor but statistically significant reductions in both D2 completion and J-maneuver rates were observed for all specialties. Comparisons between specialties found no significant differences in KPIs, with the exception of precertification J-maneuver rate $(P=0.001)$, which was highest in GS trainees ( $P=0.001$ vs. gastroenterology; $P=0.019$ vs. NME), although this was not evident post-certification $(P=$ 0.523).

\section{Flexible sigmoidoscopy}

Paired data were analyzed for $56.7 \%$ of trainees awarded certification in flexible sigmoidoscopy, which primarily comprised of NMEs (92/107, 86.0\%). Median (IQR) procedural numbers were 57 (IQR 32 -88) pre- and 56 (IQR 30-95) post-certification. There was no significant difference in PDR between specialties immediately before (mean $12.6 \% ; P=0.362$ ) and after certification (mean $12.0 \% ; P=0.670$ ). However, on subgroup analysis ( Table 3), a significant reduction in PDR was noted for GS ( $18.8 \%$ to $12.5 \% ; P=0.028$ ), although only seven GS trainees were included in this analysis.

\section{Colonoscopy (provisional)}

For provisional certification in colonoscopy, paired data were available for 749 certified trainees (67.8\%), each with a median of 31 pre-certification (IQR 21-45) and 29 (IQR 19-48) postcertification procedures. In the post-certification phase, a small overall reduction in CIR was observed across all groups $(95.2 \%$ to $93.8 \%, P<0.001)$. There was no overall difference in PDR between the pre- $(22.7 \%)$ and post-certification periods $(22.5 \%$, $P=0.979$ ), although subgroup analysis demonstrated increased 


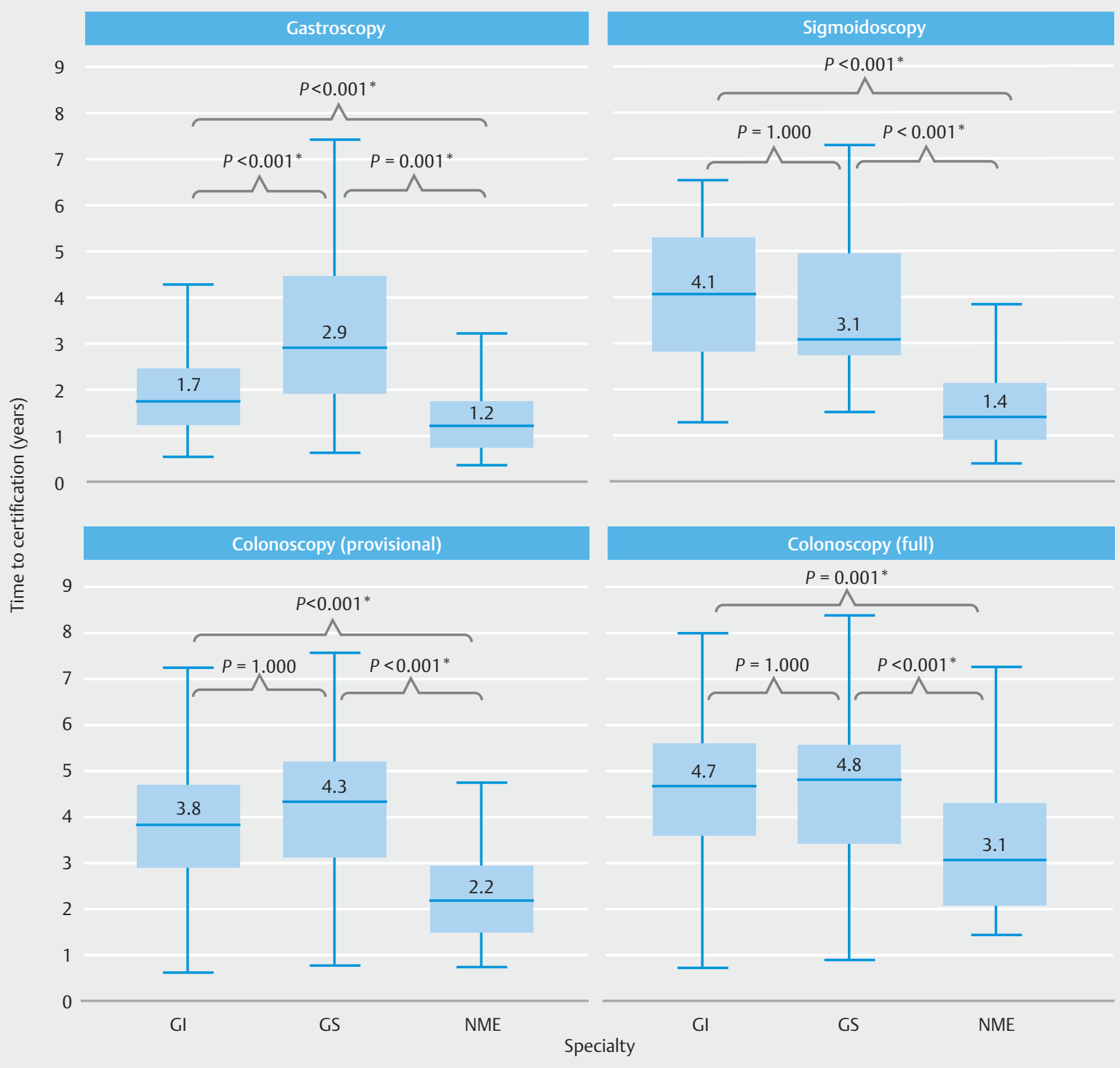

- Fig. 3 Time-to-certification (year) by specialty and modality. Excludes trainees with baseline procedures. GS: general surgeon; NME: monmedical endoscopist ${ }^{*} P<0.05$.

PDR was recorded for NMEs $(20.2 \%$ to $22.4 \%, p=0.010)$ postcertification ( $\triangleright$ Table 3 ).

\section{Colonoscopy (full)}

For full colonoscopy certification, 297 endoscopists recorded paired data (55.3\%), each with a median of 43 pre-certification (IQR 26-65) and 31 (IQR 21-48) post-certification procedures. No significant difference in CIR was detected between the periods pre- and post-certification (mean: $94.9 \%$ vs. $94.4 \%, P=0.315$ ), with subgroup analyses by specialty returning consistent results ( $\downarrow$ Table 3 ). There was no overall difference in PDR (25.6\% to $24.4 \%, P=0.075$ ), although a significant reduction was observed within the GS subgroup (24.3\% to $20.9 \%, P=0.007)$. There were no significant differences between specialty in terms of pre-certification CIR $(P=0.283)$, post-certification CIR $(P=0.771)$ or pre-certification PDR $(P=$ $0.469)$, although there was variation in post-certification PDR $(P=0.004)$, which was lowest in the GS specialty.

\section{Outcomes of applications for certification}

Outcomes of summative assessment are summarised in $>$ Table 4. Summative assessment was successful at first attempt in 91.3\%. Failure rates at first attempt varied between specialty for gastroscopy (gastroenterology $11.0 \%$, GS $10.5 \%$, NME $3.4 \% ; P=0.016$ ) and sigmoidoscopy (gastroenterology $4.6 \%$, GS 30.0\%, NME 9.3\%; $P=0.012$ ), but not provisional colonoscopy certification (gastroenterology 6.6\%, GS 5.9\%, NME $10.7 \%$; $P=0.130$ ). Analyses combining all of the procedures found no significant differences in summative assessment failure rates 
Table 2 Paired median key performance indicator data for trainees in the 3 months pre- and post-gastroscopy certification period with comparisons performed using Wilcoxon signed rank tests.

\begin{tabular}{|l|c|c|c|c|c|c|c|}
\hline \multicolumn{4}{|l|}{ Upper gastrointestinal endoscopy certification } & Mean D2 intubation Rate & \multicolumn{3}{|c|}{ Mean J-maneuver rate } \\
\hline Gastroscopy & N & Pre & Post & P value & Pre & Post \\
\hline Gastroenterologist & 560 & $97.5 \%$ & $96.4 \%$ & $<0.001$ & $97.0 \%$ & $95.8 \%$ \\
\hline GS & 101 & $97.8 \%$ & $94.7 \%$ & $<0.001$ & $98.2 \%$ & $95.5 \%$ \\
\hline NME & 85 & $97.6 \%$ & $96.7 \%$ & 0.016 & $97.7 \%$ & $96.3 \%$ \\
\hline Overall & 768 & $97.6 \%$ & $96.2 \%$ & $<0.001$ & $97.3 \%$ & $95.8 \%$ \\
\hline
\end{tabular}

Trainees with $<10$ procedures in the 3 months post-certification period were excluded. The overall numbers include data from all certified specialties. GS: gastroentestinal surgeon; NME: non-medical endoscopist.

- Table 3 Paired mean key performance indicator data for trainees in the 3 months pre- and post-lower Gl certification period, with comparisons performed using Wilcoxon signed rank tests.

\begin{tabular}{|c|c|c|c|c|c|c|c|}
\hline \multicolumn{2}{|c|}{ Lower gastrointestinal endoscopy certification } & \multicolumn{3}{|c|}{ Mean CIR } & \multicolumn{3}{|c|}{ Mean PDR } \\
\hline Sigmoidoscopy & $\mathbf{N}$ & Pre & Post & $P$ value & Pre & Post & $P$ value \\
\hline Gastroenterologist & 8 & & & & $20.3 \%$ & $20.0 \%$ & 0.345 \\
\hline GS & 7 & & & & $18.8 \%$ & $12.5 \%$ & 0.028 \\
\hline NME & 92 & & & & $12.2 \%$ & $12.0 \%$ & 0.463 \\
\hline Overall & 110 & & & & $12.6 \%$ & $12.0 \%$ & 0.674 \\
\hline Colonoscopy (provisional) & $\mathbf{N}$ & Pre & Post & $P$ value & Pre & Post & $P$ value \\
\hline Gastroenterologist & 382 & $95.4 \%$ & $94.2 \%$ & 0.001 & $22.9 \%$ & $22.7 \%$ & 0.871 \\
\hline GS & 234 & $95.1 \%$ & $93.4 \%$ & 0.008 & $23.6 \%$ & $22.1 \%$ & 0.109 \\
\hline NME & 115 & $94.6 \%$ & $93.1 \%$ & 0.004 & $20.2 \%$ & $22.4 \%$ & 0.010 \\
\hline Overall & 749 & $95.2 \%$ & $93.8 \%$ & $<0.001$ & $22.7 \%$ & $22.5 \%$ & 0.979 \\
\hline Colonoscopy (full) & $\mathbf{N}$ & Pre & Post & $P$ value & Pre & Post & $P$ value \\
\hline Gastroenterologist & 173 & $94.9 \%$ & $94.4 \%$ & 0.492 & $25.6 \%$ & $25.4 \%$ & 0.759 \\
\hline GS & 92 & $94.9 \%$ & $94.4 \%$ & 0.409 & $24.3 \%$ & $20.9 \%$ & 0.007 \\
\hline NME & 32 & $93.5 \%$ & $94.8 \%$ & 0.984 & $27.9 \%$ & $25.9 \%$ & 0.421 \\
\hline Overall & 301 & $94.9 \%$ & $94.4 \%$ & 0.315 & $25.6 \%$ & $24.4 \%$ & 0.075 \\
\hline
\end{tabular}

Table 4 Outcomes of summative assessment.

\begin{tabular}{|l|l|l|l|}
\hline $\begin{array}{l}\text { Certification } \\
\text { modality }\end{array}$ & $\begin{array}{l}\text { Pass at first } \\
\text { attempt }\end{array}$ & $\begin{array}{l}\text { Second at- } \\
\text { tempt if failed }\end{array}$ & $\begin{array}{l}\text { Pass at sec- } \\
\text { ond attempt }\end{array}$ \\
\hline Gastroscopy & $\begin{array}{l}1182 / 1312 \\
(90.1 \%)\end{array}$ & $\begin{array}{l}113 / 130 \\
(86.9 \%)\end{array}$ & $\begin{array}{l}103 / 113 \\
(91.2 \%)\end{array}$ \\
\hline Sigmoidoscopy & $\begin{array}{l}173 / 194 \\
(89.2 \%)\end{array}$ & $\begin{array}{l}15 / 21 \\
(71.4 \%)\end{array}$ & $\begin{array}{l}14 / 15 \\
(93.3 \%)\end{array}$ \\
\hline Colonoscopy & $1029 / 1105$ & $64 / 76$ & $54 / 64$ \\
\hline (provisional) & $(93.1 \%)$ & $(84.2 \%)$ & $(84.4 \%)$ \\
\hline Total & $2384 / 2611$ & $192 / 227$ & $171 / 192$ \\
\hline & $(91.3 \%)$ & $(84.6 \%)$ & $(89.1 \%)$ \\
\hline
\end{tabular}

at first attempt between specialty (gastroenterology $9.1 \%$, GS 8.6\%, NME 7.8\%; $\mathrm{p}=0.688$ ).

A total of 3183 applications for JAG certification fulfilled summative criteria and were reviewed centrally by JETS assessors. Of these, $26(0.8 \%)$ applications were turned down due to the following reasons: not meeting current criteria $(n=14)$, insufficient polypectomy experience $(n=3)$, lack of recent evidence following summative assessment $(n=3)$ and potential assessment bias, i. e. lack of variation in assessors or spread of formative assessments over time $(n=6)$. 


\section{Discussion}

This prospective study provides novel data from the JETS eportfolio related to endoscopy certification in the UK. Quality criteria laid out in the JAG accreditation standards for endoscopy units ensure that no endoscopist is allowed to perform independently without JAG certification [7], which is reliant on engagement with the e-portfolio. This offers a unique insight into the effectiveness of certification and whether important differences exist in how different specialties approach certification and perform in the independent setting thereafter.

Following national rollout of the JETS e-portfolio, JAG certification numbers have increased in parallel with increasing trainee numbers and rising endoscopy demand. The relative increase in sigmoidoscopy and colonoscopy certification is likely to reflect a response to rollout of the National 'Bowel Scope' (sigmoidoscopy screening) programme [19] and Bowel Cancer Screening Programme [20], in addition to several public symptom awareness campaigns [21,22]. Between 2011 and 2017, NMEs accounted for the largest proportional increase in certification numbers, in part owing to a government initiative to increase the flexible sigmoidoscopy workforce (NHS England NME program) [18]. Trainees from the NME specialty achieved certification in the shortest overall training time, despite logging significantly more precertification procedures than GS (for gastroscopy and colonoscopy certification) and gastroenterologists (for full colonoscopy certification). The mismatch between the number of procedures and time to certification indicates variation in access to training opportunities, intensity of training and the potential for differences in acquisition of endoscopic skills according to training specialty. UK trainee survey data suggest disparities between specialties [23,24], training grades [25], and regions [25] in terms of access to endoscopy training. Conflicting commitments with general medical oncall rotas (gastroenterology and GS) and theater-based training (GS), competition for training (with expansion of NME numbers), and a shortage of dedicated training lists are cited as contributory factors [26]. These surveys highlight the imperfections and pressures of real-world endoscopy training systems within the UK, which may be less applicable internationally. It is important to note that, with exception to gastroscopy certification in gastroenterology trainees, JAG certification is not a criterion for completion of gastroenterology and GS specialist training programs, which typically last 5 years.

For each specialty, median procedural numbers at certification exceeded the minimum thresholds set by JAG which were originally determined using procedural completion metrics $[27,28]$. As certification is a composite endpoint requiring the maturation of a range of technical and non-technical competencies which are assessed within DOPS, this study suggests a more realistic requirement of procedural numbers and training times to achieve this competence standard. It is recognized that within a cohort, trainees develop competency at different rates. Setting higher minimum procedural numbers may penalize those who develop competency at a faster pace, whereas lowering the threshold may reduce the breadth of caseload encountered by a trainee. The certification process provides a competency safeguard for those who elect to trigger summative assessment towards the minimum end of the JAG procedural threshold. Formative DOPS assessments enable trainers to monitor development of individual competencies during training [8] and to gauge readiness for summative assessment. Trainees may apply for summative DOPS assessment upon fulfilment of JAG criteria, but crucially, also requires support from the trainer and training lead to entrust them for unsupervised practice.

The effect of specialty on performance and skills acquisition during endoscopy training is unclear. Previous JETS e-portfolio analyses $[27,28]$ had suggested an association between trainee specialty and unassisted procedural completion rates. After multivariate analysis to account for factors including lifetime procedural count and training intensity, GS trainees were more likely to achieve unassisted procedural completion for gastroscopy [27] and colonoscopy [28] compared to gastroenterology and NME trainees. However, these studies involved trainees at early stages of training, using procedural completion metrics as the outcome. In contrast, our study explored differences in additional KPIs between specialties in a certified cohort who were deemed competent for independent practice. For gastroscopy, although there were post-certification reductions in rates of D2 intubation and J-maneuver, average rates remained above JAG standards. Unadjusted CIR fell after provisional colonoscopy certification but remained above the $90 \%$ national standard, with no significant changes seen after progression to full certification. The PDR in both periods remained above the $20 \%$ aspirational target set in UK guidelines [29], and comparable to the PDR of $20.3 \%$ for diagnostic colonoscopy demonstrated within the last UK colonoscopy audit [30]. Despite subtle variations, overall performance for all specialty trainees was maintained above the required JAG standards [4-6]. This suggests that JAG requirements for trainee certification are appropriate in determining subsequent competence for independent practice, defined by national quality standards and guidelines $[4-6,29,31]$. Furthermore, the failure rates of $10 \%$ during the summative phase and $0.8 \%$ following central JETS assessor review, attests to the stringency of the certification process.

Several limitations require discussion. First, our study was an exploratory evaluation of the certification cohort. It did not include or assess outcomes of trainees who had commenced endoscopy training or completed gastroenterology/GS specialist training, but not achieved certification. KPIs were limited to those recorded by the JETS e-portfolio and those included in the analysis. They did not assess procedural factors, e.g. age, procedure indication, diagnoses, bowel preparation, discomfort scores, or sedation use, or training factors, e. g. training intensity, which has been explored previously $[27,28]$. It was therefore unclear whether decrements in performance metrics, e.g. PDR in GS trainees after full colonoscopy certification, could be due to changes in caseload after certification. Second, due to the nature of the e-portfolio and e-certification roll-out, a significant number $(37.1 \%)$ of trainees had baseline procedures preceding use of the e-portfolio. Exclusion of these cases in the number of procedures needed to achieve certification analyses is a potential source of bias. It was therefore not possi- 
ble to perform accurate trend analyses for time-to-certification due to the significant proportion who had baseline endoscopy experience prior to the implementation of the e-portfolio. Third, time-to-certification does not necessarily indicate timeto-competency; trainees may defer applying for certification until they feel ready to perform independently. The interval between eligibility for certification and application for certification was not studied. Fourth, although e-portfolio entries have been compared to real-world procedure entries and shown to be reliable $[27,28]$, analyses of a trainee-maintained e-portfolio are not free from selection bias and the unintended consequences of performance monitoring. As such, several measures have been implemented within the certification process to ensure validity of the submitted data. These include use of independent assessors for summative assessments, local review by the training lead, and review of each e-portfolio by JETS assessors. Finally, not all endoscopists continued with e-portfolio participation after certification; $61.2 \%$ submitted the minimum post-certification procedures $(\geq 10)$ to allow for paired $\mathrm{KPI}$ comparisons. The issue of entry bias will be addressed through the imminent rollout of the National Endoscopy Database in April 2018 [32], which intends to auto-populate training records directly from UK endoscopy reporting systems into the JETS e-portfolio. JAG is currently reviewing certification requirements using a performance-based approach with emphasis on formative assessments and performance metrics, particularly when the latter may be assessed more reliably with the National Endoscopy Database. Such data will assist ongoing efforts to develop evidence-based training pathways with continuing review and refinement of specified elements and how these are delivered to greatest effect within the confines of other demands on trainees and trainers.

The value of JAG certification has been accepted by both medical and surgical training committees in the UK and is understood by all practicing endoscopists and trainees. Our data support an assertion that the competency-based elements that comprise the certification standard deliver competent endoscopists who can evidence their performance standard via the e-portfolio. Few other international training systems can provide this level of quality assurance to commissioners (payers) and the populations they serve.

\section{Conclusion}

The JAG has developed a national system for certification of trainees in upper and lower gastrointestinal endoscopy. The JETS e-portfolio collects standardized training data to provide real-time monitoring of progress of trainees against certification standards. Our data confirm that the certification process can be delivered for endoscopists from different speciality groups on a national basis, in a transparent and robust manner. Trainees achieving this benchmark generally maintain national quality standards during the early post-certification period of potentially independent endoscopic practice. JAG certification can therefore be considered a mark of quality in diagnostic endoscopy. Data from the JETS e-portfolio provide a firm basis to consider certification in other endoscopic modalities.

\section{Acknowledgement}

The authors would like to acknowledge JETS assessors, the JAG office and Richard Chapman from Weblogik.

\section{Competing interests}

Dr. Siau (JAG research fellow) and Mr Broughton (JAG senior accreditation manager) hold full-time roles that are funded by JAG. Dr. Anderson and Dr. Valori are directors of AnderVal Ltd, an endoscopy training company. Dr. Pullan, Dr. Beales, Dr. Wells, and Dr. Dunckley have been paid for their role as JETS assessors. Dr. Thomas-Gibson is the current chair of JAG and has received educational support in the form of course fees from Aquilant/Fuji. Dr. Haycock has received financial support from Health Education England for committee work relating to the Clinical (Non-Medical) Endoscopist Programme.

\section{References}

[1] Siau K, Hawkes ND, Dunckley P. Training in endoscopy. Curr Treat Options Gastroenterol 2018; 16: 345-361

[2] Siau K, Green JT, Hawkes ND et al. The impact of the Joint Advisory Group on Gastrointestinal Endoscopy (JAG) on endoscopy services in the UK and beyond. Frontline Gastroenterol 2019; 10: 93-106

[3] Mehta T, Dowler K, McKaig BC et al. Development and roll out of the JETS e-portfolio: a web based electronic portfolio for endoscopists. Frontline Gastroenterol 2011; 2: 35-42

[4] Joint Advisory Group on Gastrointestinal Endoscopy. JAG trainee certification process: Diagnostic gastroscopy (OGD). Available at https:// www.thejag.org.uk/Downloads/JETS\%20-\%20certification\%20for\% 20trainees/OGD\%20application\%20criteria\%20and\%20process.pdf [Accessed November 13, 2018]

[5] Joint Advisory Group onGastrointestinal Endoscopy. JAG trainee certificationprocess - Flexible Sigmoidoscopy. Available at https://www. thejag.org.uk/Downloads/JETS\%20-\%20certification\%20for\%20trainees/Flexible\%20sigmoidoscopy\%20application\%20criteria\%20and\% 20process.pdf [Accessed January 15, 2018]

[6] Joint Advisory Group on Gastrointestinal Endoscopy. JAG trainee certification process: Colonoscopy (provisional and full). Available at https://www.thejag.org.uk/Downloads/JETS\%20-\%20certification\% 20for\%20trainees/Colonoscopy\%20application\%20criteria\%20and\% 20process.pdf [Accessed January 15, 2018]

[7] JAG. Guidelines for the training, appraisal and assessment of trainees in gastrointestinal endoscopy and for the assessment of units for registration and re-registration. 2004: Available at http://www.bsg. org.uk/pdf_word_docs/jag_recommendations_2004.pdf [Accessed August 10, 2017]

[8] Siau K, Dunckley P, Valori R et al. Changes in scoring of Direct Observation of Procedural Skills (DOPS) forms and the impact on competence assessment. Endoscopy 2018; 50: $770-778$

[9] Barton JR, Corbett S, van der Vleuten CP. The validity and reliability of a Direct Observation of Procedural Skills assessment tool: assessing colonoscopic skills of senior endoscopists. Gastrointest Endosc 2012; 75: $591-597$

[10] Gupta S, Bassett P, Man R et al. Validation of a novel method for assessing competency in polypectomy. Gastrointest Endosc 2012; 75: 568-575 
[11] Joint Advisory Group on Gastrointestinal Endoscopy. Joint Advisory Group on Gastrointestinal Endoscopy (JAG) accreditation standards for endoscopy services. 2015: Available at https://www.thejag.org. uk/Downloads/Accreditation/JAG\%20accreditation\%20standards\% 20for\%20endoscopy\%20services.pdf

[12] Joint Advisory Group on Gastrointestinal Endoscopy. Formative DOPS: Diagnostic upper gastrointestinal endoscopy (OGD). 2016: Available at https://www.thejag.org.uk/Downloads/DOPS\%20forms\%20(international\%20and\%20reference\%20use\%20only)/Formative\%20DOPS_Upper\%20G1\%20(OGD).pdf [Accessed November 13, 2018]

[13] Joint Advisory Group on Gastrointestinal Endoscopy. Formative DOPS: Colonoscopy and Flexible Sigmoidoscopy. 2016: Available at https:// www.thejag.org.uk/Downloads/DOPS\%20forms\%20(international\% 20and\%20reference\%20use\%20only)/Formative\%20DOPS_Colonoscopy\%20and\%20Flexible\%20sigmoidoscopy.pdf [Accessed November 13, 2018]

[14] Joint Advisory Group on Gastrointestinal Endoscopy. Formative DOPyS: Colonoscopy and Flexible Sigmoidoscopy. 2016: Available at https://www.thejag.org.uk/Downloads/DOPS\%20forms\%20(international\%20and\%20reference\%20use\%20only)/Formative\%20DOPyS_Colonoscopy\%20and\%20Flexible\%20sigmoidoscopy.pdf [Accessed November 13, 2018]

[15] Siau K, Hodson J, Valori RM et al. Performance indicators in colonoscopy after certification for independent practice: outcomes and predictors of competence. Gastrointest Endosc 2018: doi:10.1016/j. gie.2018.07.032

[16] Siau K, Anderson J, Beales I et al. PTU-013 Trends in certification for gastrointestinal endoscopy and variations between trainee specialties: results from the UK JETS database. Gut 2017; 66: A57

[17] Brown H, Wyatt S, Croft S et al. Scoping the Future, An evaluation of endoscopy capacity across the NHS in England. 2015: Available at https://www.thejag.org.uk/Downloads/General/scoping_the_future_-_final.pdf [Accessed February 11, 2018]

[18] Office for Public Management. Accelerated Non-Medical Endoscopist Training Programme. Report to Health Education England 2017: Available at https://traverse.Itd/application/files/1215/2044/4540/ OPM_NME-Year-1-Evaluation-Report-Final.pdf [Accessed November 13, 2018]

[19] McGregor LM, Bonello B, Kerrison RS et al. Uptake of bowel scope (flexible sigmoidoscopy) screening in the English National Programme: the first 14 months. J Med Screen 2016; 23: $77-82$
[20] Logan R, Patnick J, Nickerson C et al. Outcomes of the Bowel Cancer Screening Programme (BCSP) in England after the first 1 million tests. Gut 2012; 61: 1439-1446

[21] Siau K, Yew AC, Hingley S et al. The 2015 upper gastrointestinal "Be Clear on Cancer" campaign: its impact on gastroenterology services and malignant and premalignant diagnoses. Frontline Gastroenterol 2017; 8: 284-289

[22] Peacock O, Clayton S, Atkinson F et al. 'Be Clear on Cancer': the impact of the UK National Bowel Cancer Awareness Campaign. Colorectal Dis 2013; 15: $963-967$

[23] Jones R, Stylianides N, Robertson A et al. National survey on endoscopy training in the UK. Ann R Coll Surg Engl 2015; 97: 386- 389

[24] Leyden JE, Doherty GA, Hanley A et al. Quality of colonoscopy performance among gastroenterology and surgical trainees: A need for common training standards for all trainees? Endoscopy 2011; 43: $935-940$

[25] Bhatt S, Ambrose T, Dunckley P et al. Trainee endoscopic procedures by deanery and grade in the United Kingdom: September 2013-August 2014. Have we improved? Gut 2015; 64: A403

[26] Hammond JS, Watson NFS, Lund JN et al. Surgical endoscopy training: The Joint Advisory Group on gastrointestinal endoscopy national review. Frontline Gastroenterol 2013; 4: 20 - 24

[27] Ward S, Hancox A, Mohammed $M$ et al. The learning curve to achieve satisfactory completion rates in upper $\mathrm{Gl}$ endoscopy: an analysis of a national training database. Gut 2017; 66: 1022 - 1033

[28] Ward S, Mohammed M, Walt R et al. An analysis of the learning curve to achieve competency at colonoscopy using the JETS database. Gut 2014; 63: 1746 - 1754

[29] Rees C, Thomas Gibson S, Rutter M et al. UK key performance indicators and quality assurance standards for colonoscopy. Gut 2016; 65: $1923-1929$

[30] Gavin DR, Valori RM, Anderson JT et al. The national colonoscopy audit: a nationwide assessment of the quality and safety of colonoscopy in the UK. Gut 2013; 62: $242-249$

[31] Beg S, Ragunath K, Wyman A et al. Quality standards in upper gastrointestinal endoscopy: a position statement of the British Society of Gastroenterology (BSG) and Association of Upper Gastrointestinal Surgeons of Great Britain and Ireland (AUGIS). Gut 2017; 66: 1886 1899

[32] Lee TJW, Macdougall L, Broughton R et al. PTH-044 The National Endoscopy Database (NED) Project. Gut 2016; 65: A240 